ESAIM: PROCEEDINGS, September 2005, Vol.14, 115-123

Eric Cancès \& Jean-Frédéric Gerbeau, Editors

DOI: $10.1051 /$ proc:2005010

\title{
A PENALTY METHOD FOR THE SIMULATION OF FLUID - RIGID BODY INTERACTION
}

\author{
João Janela ${ }^{1}$, Aline Lefebvre ${ }^{2}$ and Bertrand Maury ${ }^{2}$
}

\begin{abstract}
We present here a method to simulate the motion of a rigid body in a fluid. The method is based on a variational formulation on the whole fluid/solid domain, with some constraints on the unknown and the test functions. These constraints are relaxed by introducing a penalty term, which leads to a minimization problem over unconstrained functional spaces. This makes the method straightforward to implement from any finite element Stokes/Navier-Stokes solver. It is shown that, as the penalty parameter goes to infinity, we recover the coupled fluid-solid equations. We apply this approach to a simplified $2 \mathrm{D}$ model of the aortic valve.
\end{abstract}

Résumé. Nous présentons ici une méthode de simulation du mouvement d'un corps rigide dans un fluide. Cette méthode est basée sur une formulation variationnelle sur tout le domaine fluide/solide, avec des contraintes sur l'inconnue et sur les fonctions test. On relaxe ces contraintes en introduisant un terme de pénalisation, qui conduit à un problème de minimisation sur des espaces fonctionnels non contraints. Ainsi, tout solveur éléments finis pour Stokes/Navier Stokes permet de programmer aisément cette méthode. On montre que, quand le paramètre de pénalisation tend vers l'infini, on retrouve le système d'équations couplées fluide/solide. Cette approche est appliquée à un modèle 2D simplifié de la valve aortique.

\section{INTRODUCTION}

We consider a connected bounded, regular domain $\Omega \subset \mathbb{R}^{2}$ and we denote by $B$ a subset of $\Omega$ strongly contained in $\Omega$. We shall restrict ourselves to the case where $B$ is connected (see figure 1 ), but one can easily generalize to any domain $B$ with several connected components. We suppose that $\Omega \backslash \bar{B}$ is filled with a Newtonian fluid governed by the Navier-Stokes equations and that $B$ is a rigid inclusion in $\Omega$. We suppose that $B$ is attached at one of its points $\mathbf{x}_{0}$. We shall apply our approach to a situation where the rotating rigid body is submitted to an angular pull-back moment.

Numerical simulations of such a problem can be carried out in many different ways, which we may classify into two main classes. The first one relies on a moving mesh which fits the moving part of the boundary (see e.g. [4], [5], and [7]). In the second approach, the whole domain is covered by a static mesh. Those methods are known as fictitious domain or embedded domain methods, as the actual computational domain (the fluid component) is extended to a larger domain which covers the area of interest (zones which are likely to be occupied by the fluid). To our knowledge, all fictitious domain approaches which have been applied to problems like the one we consider are based on Lagrange multipliers (see [3]), which enforce the velocity in the solid part

\footnotetext{
1 CEMAT, Instituto Superior Técnico and Departamento de Matemática, ISEG, Lisbon, Portugal;

e-mail: jjanela@iseg.utl.pt

2 Laboratoire de Mathématiques, Université Paris Sud, Orsay, France;

e-mail: Aline.Lefebvre@math.u-psud.fr \& Bertrand.Maury@math.u-psud.fr
}

(c) EDP Sciences, SMAI 2005 


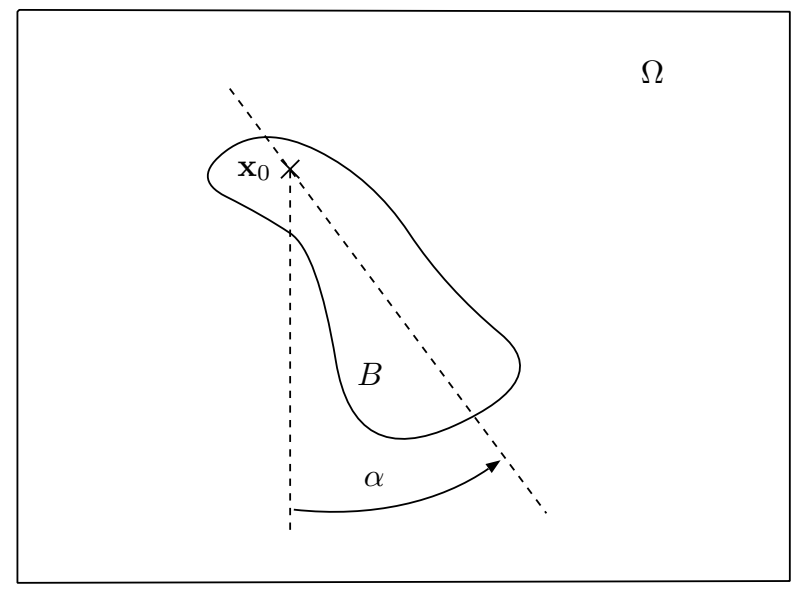

Figure 1. Model problem

to identify to the velocity of the rigid body. We propose here a penalty method to handle the rigid motion. As the penalty changes the stiffness operator, we loose an advantage of the Lagrange multiplier method, which is the possibility to use Fast Fourier Transform-like solvers. On the other hand, this method can be implemented straightforwardly on a general Finite Element solver like FreeFem ++ (see [1]), which we used to run numerical experiments.

\section{Continuous problem and Variational Formulation}

For the sake of simplicity, we will consider here homogeneous Dirichlet conditions on $\partial \Omega$. The fluid obeys Navier-Stokes equations in $\Omega \backslash \bar{B}=\Omega \backslash \bar{B}(t)$ at every time $t$, and the body motion follows the Newton law, which reduces here to an equation on the angular velocity around $\mathbf{x}_{0}$. Those equations are coupled by hydrodynamic forces exerted by the fluid on the solid. Finally, viscosity imposes no-slip conditions on the boundary of $B$ : at each point of $\partial B$, the velocity on the fluid side is equal to the velocity on the rigid side.

We denote by $\omega=\dot{\theta}$ the angular velocity of $B$, so that we have to find a velocity field $\mathbf{u}=\left(u_{1}, u_{2}\right)$ defined in $\Omega \backslash \bar{B}, \omega \in \mathbb{R}$ and a pressure field p defined in $\Omega \backslash \bar{B}$ such that :

$$
\left\{\begin{array}{rlrl}
\rho_{f} \frac{\mathrm{D} \mathbf{u}}{\mathrm{D} t}-\mu \triangle \mathbf{u}+\nabla \mathrm{p} & =\mathbf{f}_{f} & & \text { in } \Omega \backslash \bar{B} \\
\nabla \cdot \mathbf{u} & =0 & & \text { in } \Omega \backslash \bar{B} \\
\mathbf{u} & =0 & & \text { on } \partial \Omega \\
\mathbf{u}(\mathbf{x}) & =\omega\left(\mathbf{x}-\mathbf{x}_{0}\right)^{\perp} & & \text { on } \partial B \\
\mathrm{~J}_{\mathbf{x}_{0}} \dot{\omega} & =\int_{B}\left(\mathbf{x}-\mathbf{x}_{0}\right)^{\perp} \cdot \mathbf{f}_{B}-\int_{\partial B}\left(\mathbf{x}-\mathbf{x}_{0}\right)^{\perp} \cdot \sigma \mathbf{n} d s &
\end{array}\right.
$$

where $\mathbf{f}_{f}$ and $\mathbf{f}_{B}$ are the external forces exerted on the fluid and the rigid body respectively, $\rho_{f}$ and $\rho_{B}$ are their respective densities, $\mu$ is the viscosity of the fluid, $\mathbf{n}$ is the external normal to $\Omega \backslash \bar{B}, \sigma$ is the Cauchy stress tensor, $\mathrm{J}_{\mathbf{x}_{0}}$ is the kinetic momentum of $B$ at point $\mathbf{x}_{0}$ and $D \mathbf{u} / D t$ is the total derivative of $\mathbf{u}$ :

$$
\sigma=2 \mu \mathbf{D}(\mathbf{u})-\text { pId } \quad \text { and } \quad \mathbf{D}(\mathbf{u})=\frac{\nabla \mathbf{u}+(\nabla \mathbf{u})^{T}}{2},
$$




$$
\begin{aligned}
& \mathrm{J}_{\mathbf{x}_{0}}=\int_{B} \rho_{B}\left|\mathbf{x}-\mathbf{x}_{0}\right|^{2}, \\
& \frac{\mathrm{D} \mathbf{u}}{\mathrm{D} t}=\frac{\partial \mathbf{u}}{\partial t}+(\mathbf{u} \cdot \nabla) \mathbf{u},
\end{aligned}
$$

and $\mathbf{x}^{\perp}$ denotes $\left(-x_{2}, x_{1}\right)$.

Our first step will consist in establishing a variational formulation easily tractable from the numerical point of view, i.e. involving functions which are defined on the whole domain $\Omega$. This can be achieved by prescribing the constraints on both the unknown velocity field and its test counterpart, at (almost) every time. In what follows, we consider the problem at a given time $t$, and we drop the dependence of the domain $\Omega \backslash \bar{B}$ upon $t$, in order to alleviate notations. We introduce the following spaces:

$$
\begin{gathered}
K_{\mathbf{x}_{0}}=\left\{\mathbf{u} \in H_{0}^{1}(\Omega)^{2}, \int_{D} \mathbf{u}=0\right\}, K_{\mathbf{x}_{0}, \nabla}=\left\{\mathbf{u} \in K_{\mathbf{x}_{0}}, \nabla \cdot \mathbf{u}=0\right\}, \\
K_{B}=\left\{\mathbf{u} \in H_{0}^{1}(\Omega)^{2}, \exists(\mathbf{V}, \omega) \in \mathbb{R}^{2} \times \mathbb{R} \text { s.t. } \mathbf{u}=\mathbf{V}+\omega\left(\mathbf{x}-\mathbf{x}_{0}\right)^{\perp} \text { a.e. in } B\right\},
\end{gathered}
$$

where $D$ is a disc included in $B$ and centered at $\mathbf{x}_{0}$. As for $K_{B}$, which is the space of velocity fields which do not deform $B$, it can be written

$$
K_{B}=\left\{\mathbf{u} \in H_{0}^{1}(\Omega)^{2}, \mathbf{D}(\mathbf{u})=0 \text { a.e. on } B\right\} .
$$

Note that $K_{B}$ depends on the position of $B$, and therefore it is likely to vary over time. For the sake of clarity, we shall occasionally denote an element of $K_{B}$ by expliciting the real degrees of freedom: $\mathbf{U}=(\mathbf{u}, \mathbf{V}, \omega) \in K_{B}$. Note that if $\mathbf{U}=(\mathbf{u}, \mathbf{V}, \omega) \in K_{B} \cap K_{\mathbf{x}_{0}}$ then $\mathbf{V}$ is necessarily equal to zero, which expresses the fact that $B$ is fixed at $\mathbf{x}_{0}$. We nevertheless keep $\mathbf{V}$ as an unknown, because both contraints will be dealt with in different ways in actual computations. The subspaces we have introduced are closed in $H_{0}^{1}(\Omega)^{2}$ and they are consequently Hilbert spaces for the $H^{1}$ norm. Let now $\mathbf{U}=(\mathbf{u}, \mathbf{V}, \omega) \in K_{\mathbf{x}_{0}} \cap K_{B}$ be a solution of the problem at a certain time $t$. We multiply the Navier Stokes equation by $\tilde{\mathbf{U}}=(\tilde{\mathbf{u}}, \tilde{\mathbf{V}}, \tilde{\omega}) \in K_{\mathbf{x}_{0}} \cap K_{B}$ and integrate it over $\Omega \backslash \bar{B}$ :

$$
\int_{\Omega \backslash \bar{B}} \rho_{f} \frac{\mathrm{Du}}{\mathrm{D} t} \cdot \tilde{\mathbf{u}}-\mu \int_{\Omega \backslash \bar{B}} \Delta \mathbf{u} \cdot \tilde{\mathbf{u}}+\int_{\Omega \backslash \bar{B}} \nabla \mathrm{p} \cdot \tilde{\mathbf{u}}=\int_{\Omega \backslash \bar{B}} \mathbf{f}_{f} \cdot \tilde{\mathbf{u}} .
$$

Integration by parts gives

$$
\int_{\Omega \backslash \bar{B}} \rho_{f} \frac{\mathrm{D} \mathbf{u}}{\mathrm{D} t} \cdot \tilde{\mathbf{u}}+2 \mu \int_{\Omega \backslash \bar{B}} \mathbf{D}(\mathbf{u}): \mathbf{D}(\tilde{\mathbf{u}})-\int_{\Omega \backslash \bar{B}} \mathrm{p} \nabla \cdot \tilde{\mathbf{u}}-\int_{\partial(\Omega \backslash \bar{B})} \sigma \mathbf{n} \cdot \tilde{\mathbf{u}}=\int_{\Omega \backslash \bar{B}} \mathbf{f}_{f} \cdot \tilde{\mathbf{u}} .
$$

Then, using the fact that $\tilde{\mathbf{u}}=\tilde{\omega}\left(\mathbf{x}-\mathbf{x}_{0}\right)^{\perp}$ in $B$ and using the boundary conditions on $\partial B$, we obtain :

$$
\int_{\Omega \backslash \bar{B}} \rho_{f} \frac{\mathrm{D} \mathbf{u}}{\mathrm{D} t} \cdot \tilde{\mathbf{u}}+\mathrm{J}_{\mathbf{x}_{0}} \dot{\omega} \tilde{\omega}+2 \mu \int_{\Omega \backslash \bar{B}} \mathbf{D}(\mathbf{u}): \mathbf{D}(\tilde{\mathbf{u}})-\int_{\Omega \backslash \bar{B}} \mathrm{p} \nabla \cdot \tilde{\mathbf{u}}=\int_{\Omega \backslash \bar{B}} \mathbf{f}_{f} \cdot \tilde{\mathbf{u}}+\int_{B} \mathbf{f}_{B} \cdot \tilde{\mathbf{u}} .
$$

Since $\tilde{\mathbf{u}} \in K_{B}$, the third term can be written over $\Omega$ and, since $\mathbf{D}(\mathbf{u})=0$ implies $\nabla \cdot \mathbf{u}=0$, so can be the fourth one :

$$
\int_{\Omega \backslash \bar{B}} \rho_{f} \frac{\mathrm{Du}}{\mathrm{D} t} \cdot \tilde{\mathbf{u}}+\mathrm{J}_{\mathbf{x}_{0}} \dot{\omega} \tilde{\omega}+2 \mu \int_{\Omega} \mathbf{D}(\mathbf{u}): \mathbf{D}(\tilde{\mathbf{u}})-\int_{\Omega} \mathrm{p} \nabla \cdot \tilde{\mathbf{u}}=\int_{\Omega} \mathbf{f} \cdot \tilde{\mathbf{u}}
$$

where $\mathbf{f}=\mathbf{f}_{f} \chi_{\Omega \backslash \bar{B}}+\mathbf{f}_{B} \chi_{B}$. Finally, using that $\mathbf{u}=\omega\left(\mathbf{x}-\mathbf{x}_{0}\right)^{\perp}$ and $\tilde{\mathbf{u}}=\tilde{\omega}\left(\mathbf{x}-\mathbf{x}_{0}\right)^{\perp}$ in $B$ we can prove that

$$
\mathrm{J}_{\mathbf{x}_{0}} \dot{\omega} \tilde{\omega}=\int_{B} \rho_{B} \dot{\omega} \tilde{\omega}\left|\mathbf{x}-\mathbf{x}_{0}\right|^{2}=\int_{B} \rho_{B} \frac{\mathrm{Du}}{\mathrm{D} t} \cdot \tilde{\mathbf{u}}
$$


which leads to the variational formulation

$$
\left\{\begin{array}{l}
\int_{\Omega} \rho \frac{\mathrm{Du}}{\mathrm{D} t} \cdot \tilde{\mathbf{u}}+2 \mu \int_{\Omega} \mathbf{D}(\mathbf{u}): \mathbf{D}(\tilde{\mathbf{u}})-\int_{\Omega} \mathrm{p} \nabla \cdot \tilde{\mathbf{u}}=\int_{\Omega} \mathbf{f} \cdot \tilde{\mathbf{u}} \quad \forall \tilde{\mathbf{u}} \in K_{\mathbf{x}_{0}} \cap K_{B} \\
\int_{\Omega} \mathrm{q} \nabla \cdot \mathbf{u}=0 \quad \forall \mathrm{q} \in L^{2}(\Omega)
\end{array}\right.
$$

where $\rho=\rho_{f} \chi_{\Omega \backslash \bar{B}}+\rho_{B} \chi_{B}$.

\section{Time discRetization And Penalty Method}

\subsection{Time discretization}

We use the method of characteristics to discretize the total derivative. Note that, as $\rho$ is constant along trajectories, we have

$$
\rho \frac{\mathrm{Du}}{\mathrm{D} t}=\frac{\mathrm{D} \rho \mathbf{u}}{\mathrm{D} t}
$$

We denote by $\mathbf{X}^{n}(\mathbf{x})$ an approximation of $\mathbf{X}(\mathbf{x},(n+1) \Delta t, n \Delta t)$ where $\mathbf{X}$ is the characteristic associated to $\mathbf{u}$. It can be expressed as the solution to the following problem:

$$
\left\{\begin{array}{l}
\frac{\partial \mathbf{X}}{\partial \tau}(\mathbf{x}, t, \tau)=\mathbf{u}(\mathbf{X}(\mathbf{x}, t, \tau), \tau) \\
\mathbf{X}(\mathbf{x}, t, t)=\mathbf{x}
\end{array}\right.
$$

So, the time discretized problem is written at each time step :

$$
\left\{\begin{array}{rl}
\mathbf{u}^{n+1} \in K_{\mathbf{x}_{0}} \cap K_{B^{n+1}} \text { and } \mathrm{p}^{n+1} \in L^{2}(\Omega) & \\
\alpha \int_{\Omega} \rho^{n+1} \mathbf{u}^{n+1} \cdot \tilde{\mathbf{u}}+2 \mu \int_{\Omega} \mathbf{D}\left(\mathbf{u}^{n+1}\right): \mathbf{D}(\tilde{\mathbf{u}})-\int_{\Omega} \mathrm{p}^{n+1} \nabla \cdot \tilde{\mathbf{u}} & \\
= & \alpha \int_{\Omega}\left(\rho^{n} \mathbf{u}^{n}\right) \circ \mathbf{X}^{n} \cdot \tilde{\mathbf{u}}+\int_{\Omega} \mathbf{f}^{n+1} \cdot \tilde{\mathbf{u}} \quad \\
\int_{\Omega} \mathrm{q} \nabla \cdot \mathbf{u}^{n+1}=0 & \forall \mathrm{q} \in L^{2}(\Omega),
\end{array} \quad \forall \tilde{\mathbf{u}} \in K_{\mathbf{x}_{0}} \cap K_{B^{n+1}},\right.
$$

where $\alpha=1 / \Delta t$ and $B^{n+1}$ is computed using $\theta^{n+1}=\theta^{n}+\Delta t \omega^{n}$.

\subsection{Penalty method}

The previous formulation involves test functions in the constrained space of rigid motions on $B$. In order to relax this constraint, and make the algorithm easily implementable, we propose here a penalty method. Note that the rigid motion constraint can also be taken into account by mean of tensorial Lagrange multipliers, as it is done in $[9]$.

Firstly, we write problem (3) as a minimization problem:

$$
\left\{\begin{array}{l}
\mathbf{u}^{n+1} \in K_{\mathbf{x}_{0}, \nabla} \cap K_{B^{n+1}} \\
\mathrm{~J}^{n}\left(\mathbf{u}^{n+1}\right)=\min _{\mathbf{v} \in K_{\mathbf{x}_{0}, \nabla} \cap K_{B^{n+1}}} \mathrm{~J}^{n}(\mathbf{v})
\end{array}\right.
$$

where

$$
\mathbf{J}^{n}(\mathbf{v})=\frac{\alpha}{2} \int_{\Omega} \rho^{n+1} \mathbf{v}^{2}+\mu \int_{\Omega} \mathbf{D}(\mathbf{v}): \mathbf{D}(\mathbf{v})-\alpha \int_{\Omega}\left(\rho^{n} \mathbf{u}^{n}\right) \circ \mathbf{X}^{n} \cdot \mathbf{v}-\int_{\Omega} \mathbf{f}^{n+1} \cdot \mathbf{v}
$$


Problems (3) and (4) are equivalent, in the sense that $\left(\mathbf{u}^{n+1}, \mathrm{p}^{n+1}\right)$ solves (3) implies that $\mathbf{u}^{n+1}$ is a solution to (4), and if $\mathbf{u}^{n+1}$ is a solution to (4), there exists $\mathrm{p}^{n+1}$ such that $\left(\mathbf{u}^{n+1}, \mathrm{p}^{n+1}\right)$ is a solution to (3). Uniqueness of the pressure is of course out of reach, as it is clearly underdetermined within the rigid body. Existence and uniqueness of a solution to (4) is a direct consequence of Korn's second inequality (see [8]) and Lax Milgram theorem.

We are now going to approach that minimization problem with another minimization problem by penalizing the rigid motion constraint:

where

$$
\left\{\begin{array}{l}
\mathbf{u}_{\epsilon}^{n+1} \in K_{\mathbf{x}_{0}, \nabla} \\
\mathrm{J}_{\epsilon}^{n}\left(\mathbf{u}_{\epsilon}^{n+1}\right)=\min _{\mathbf{v} \in K_{\mathbf{x}_{0}, \nabla}} \mathrm{J}_{\epsilon}^{n}(\mathbf{v})
\end{array}\right.
$$

$$
\mathrm{J}_{\epsilon}^{n}(\mathbf{v})=\mathrm{J}^{n}(\mathbf{v})+\frac{1}{\epsilon} \int_{B^{n+1}} \mathbf{D}(\mathbf{v}): \mathbf{D}(\mathbf{v})
$$

Note that, as before, we can prove that problem (5) admits a unique solution.

\subsection{Convergence of the penalty method}

We establish here the convergence of the penalty method at each time step. To that purpose, we introduce the following abstract framework. We denote by $V$ a Hilbert space, $a$ and $b$ two bilinear, symmetric and continuous forms on $V, a$ being coercive and $b$ being positive $(b(\mathbf{u}, \mathbf{u}) \geq 0)$, and $\phi$ a linear form on $V$. We consider the following problems :

and

$$
\left\{\begin{array}{l}
\mathbf{u} \in\{\mathbf{w} \in V \text { s.t. } b(\mathbf{w}, \mathbf{w})=0\} \\
\mathrm{J}(\mathbf{u})=\min _{\mathbf{v} \in\{\mathbf{w} \in V \text { s.t. } b(\mathbf{w}, \mathbf{w})=0\}} \mathrm{J}(\mathbf{v})
\end{array}\right.
$$

where

$$
\left\{\begin{array}{l}
\mathbf{u}_{\epsilon} \in V \\
\mathrm{~J}_{\epsilon}\left(\mathbf{u}_{\epsilon}\right)=\min _{\mathbf{v} \in V} \mathrm{~J}_{\epsilon}(\mathbf{v})
\end{array}\right.
$$

It is shown in $[6]$ that :

$$
\mathrm{J}(\mathbf{v})=\frac{1}{2} a(\mathbf{v}, \mathbf{v})-\langle\phi, \mathbf{v}\rangle, \mathrm{J}_{\epsilon}(\mathbf{v})=\mathrm{J}(\mathbf{v})+\frac{1}{\epsilon} b(\mathbf{v}, \mathbf{v})
$$

Theorem 1. If $\mathbf{u}$ and $\mathbf{u}_{\epsilon}$ are respectively solution to problem (6) and problem ( 7 ) then $\mathbf{u}_{\epsilon}$ tends (strongly) to $\mathbf{u}$ as $\epsilon$ goes to 0 . Moreover, if $b$ can be written $b(\mathbf{u}, \mathbf{v})=(\Psi \mathbf{u}, \Psi \mathbf{v})_{\Gamma}$ where $\Gamma$ is a Hilbert space and $\Psi$ is a continuous linear mapping from $V$ to $\Gamma$, with closed range, we have the following error estimate :

$$
\exists C>0 \text { s.t. }\left|\mathbf{u}_{\epsilon}-\mathbf{u}\right| \leq C \epsilon
$$

Our problem fits into this framework, with (up to multiplicative constants)

$$
\begin{aligned}
& V=K_{\mathbf{x}_{0}, \nabla} \\
& a(\mathbf{u}, \mathbf{v})=\int_{\Omega} \mathbf{u} \cdot \mathbf{v}+2 \mu \int_{\Omega} \mathbf{D}(\mathbf{u}): \mathbf{D}(\mathbf{v}) \\
& b(\mathbf{u}, \mathbf{v})=\int_{B} \mathbf{D}(\mathbf{u}): \mathbf{D}(\mathbf{v})
\end{aligned}
$$

The linear convergence in $\epsilon$ is then a consequence of the following lemma 
Lemma 1. The mapping $\left.\mathbf{u} \longmapsto \mathbf{D}(\mathbf{u})\right|_{B}$ is linear and continuous from $K_{\mathbf{x}_{0}, \nabla}$ to $\left(L^{2}(B)\right)^{4}$, and its range is closed.

Proof. We consider a sequence $\left(\mathbf{u}_{n}\right)_{n \in \mathbb{N}} \in K_{\mathbf{x}_{0}, \nabla}$ such that $\left.\mathbf{D}\left(\mathbf{u}_{n}\right)\right|_{B}$ tends to $z$ in $\left(L^{2}(B)\right)^{4}$ as $n$ goes to infinity. We are going to build $\mathbf{u} \in K_{\mathbf{x}_{0}, \nabla}$ such that $z=\left.\mathbf{D}(\mathbf{u})\right|_{B}$. We consider $\overline{\mathbf{u}}_{n} \in K_{\mathbf{x}_{0}, \nabla}(B)$, the orthogonal projection of $\left.\mathbf{u}_{n}\right|_{B}$ on $\left(K_{\mathbf{x}_{0}, \nabla}(B) \cap K_{B}\right)^{\perp}$. Since $\overline{\mathbf{u}}_{n}$ is orthogonal to the space of rigid motions on $B$ we have (see [8]) :

$$
\left\|\overline{\mathbf{u}}_{n}\right\|_{H^{1}(B)} \leq C\left\|\mathbf{D}\left(\overline{\mathbf{u}}_{n}\right)\right\|_{L^{2}(B)}
$$

As $\mathbf{D}\left(\overline{\mathbf{u}}_{n}\right)=\left.\mathbf{D}\left(\mathbf{u}_{n}\right)\right|_{B}$, it follows that $\left(\overline{\mathbf{u}}_{n}\right)_{n}$ is bounded in $H^{1}(B)$. We now want to extend $\overline{\mathbf{u}}_{n}$ over $\Omega$. Since $\int_{\partial B} \overline{\mathbf{u}}_{n} \cdot \mathbf{n}=0$, we can construct (see [2]) a divergence free extension on $\Omega$ bounded in $H^{1}(\Omega)$ by $\left\|\overline{\mathbf{u}}_{n}\right\|_{H^{1}(B)}$. Up to an extracted subsequence, this last sequence converges weakly to $\mathbf{u} \in K_{\mathbf{x}_{0}, \nabla}$ as $\mathrm{n}$ goes to infinity and it follows immediately that $z=\left.\mathbf{D}(\mathbf{u})\right|_{B}$.

\section{NUMERICAL RESULTS}

In this section we describe how the penalty method introduced in section 2.3 can be used to simulate the motion of an idealized aortic valve.

\subsection{Description of the model}

In this somewhat over-simplified model, the "valve" is supposed to be rigid. We furthermore assume it is rotating around $\mathbf{x}_{0}$, center of $D_{1}$. The geometry outlined in Figure 2 arises from some geometric simplifications of the real physical geometry of the aortic valve that can be found in [10]. The elastic complex behaviour that

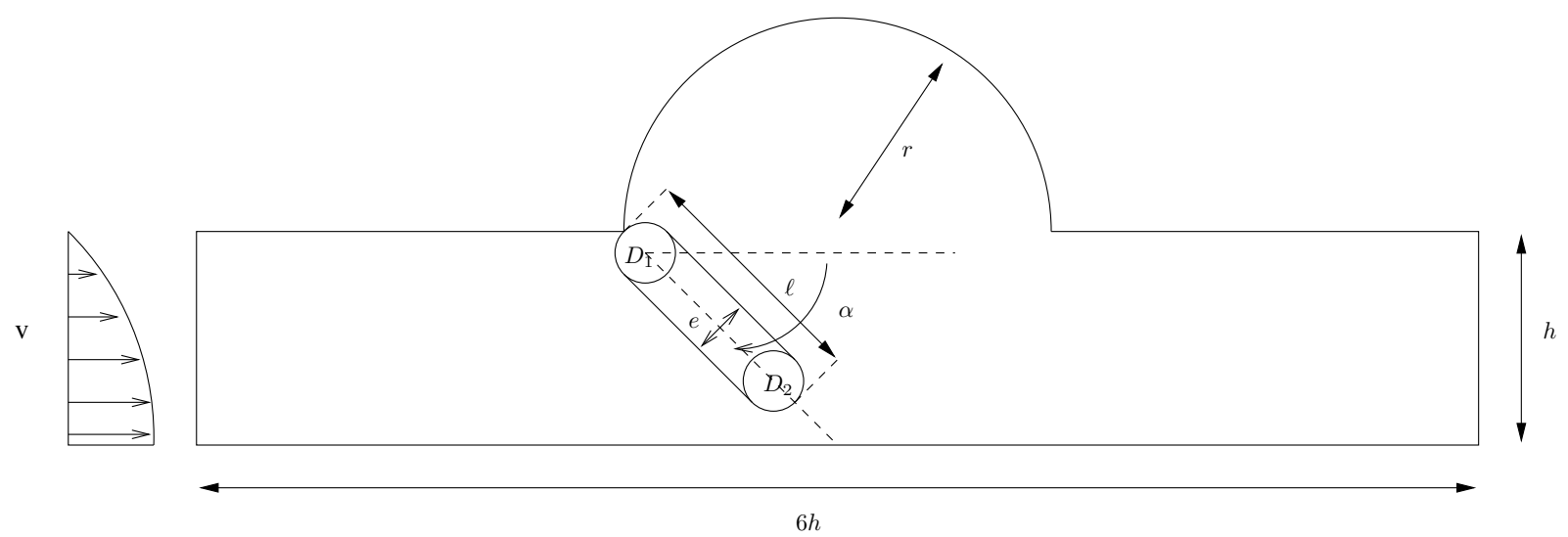

FiguRE 2. Geometry of the model problem

makes the valve return to an equilibrium position has been modeled adding a pull-back moment. More precisely, we added an external force term, acting on $D_{2}$, whose moment is proportionnal to $\alpha-\alpha_{e q}$ where $\alpha_{e q}$ is the angle of equilibrium. Therefore, the external force term including gravity and pull-back moment is :

$$
\mathbf{f}_{1}=\frac{C}{\ell}\left(\alpha-\alpha_{e q}\right) \sin \alpha \chi_{D_{2}}, \quad \mathbf{f}_{2}=\left(\rho_{f}-\rho_{s}\right) \chi_{B}-\frac{C}{\ell}\left(\alpha-\alpha_{e q}\right) \cos \alpha \chi_{D_{2}} .
$$

where $\mathrm{C}$ is a constant.

As for boundary conditions we have prescribed a pulsatile Poiseuille-type velocity profile on the left-hand side of the boundary, natural outlet conditions on the right-hand side, no-slip conditions on the top boundary and symmetry conditions on the bottom boundary. 


\subsection{Variational Formulation, implementation and results}

We use the variational formulation associated with the minimization problem (5), that reads for each time step

$$
\left\{\begin{aligned}
\mathbf{u}^{n+1} \in K_{\mathbf{x}_{0}} \text { and } \mathrm{p}^{n+1} \in L^{2}(\Omega) & \\
\alpha \int_{\Omega} \rho^{n+1} \mathbf{u}^{n+1} \cdot \tilde{\mathbf{u}} & +2 \mu \int_{\Omega} \mathbf{D}\left(\mathbf{u}^{n+1}\right): \mathbf{D}(\tilde{\mathbf{u}})+\frac{2}{\epsilon} \int_{B^{n+1}} \mathbf{D}\left(\mathbf{u}^{n+1}\right): \mathbf{D}(\tilde{\mathbf{u}})-\int_{\Omega} \mathrm{p}^{n+1} \nabla \cdot \tilde{\mathbf{u}} \\
& =\alpha \int_{\Omega}\left(\rho^{n} \mathbf{u}^{n}\right) \circ \mathrm{X}^{n} \cdot \tilde{\mathbf{u}}+\int_{\Omega} \mathbf{f}^{n+1} \cdot \tilde{\mathbf{u}} \quad \forall \tilde{\mathbf{u}} \in K_{\mathbf{x}_{0}}, \\
\int_{\Omega} \mathrm{q} \nabla \cdot \mathbf{u}^{n+1}=0 \quad \forall \mathrm{q} \in L^{2}(\Omega), &
\end{aligned}\right.
$$

The only constraint still present in the functional spaces is the one related to the existence of a fixed point in $B$ for all time, i.e. $\mathbf{u}^{n+1} \in K_{\mathbf{x}_{0}}$.

One way of enforcing this condition is to look for solutions satisfying $\int_{D_{1}} \mathbf{u}=0$. Even though we could enforce this condition by penalization, there would be terms in the corresponding variational formulation that cannot be handled easily by standard solvers. So we enforce the zero mean velocity condition by duality. This amounts to add the extra term $\int_{D_{1}} \boldsymbol{\lambda} \cdot \tilde{\mathbf{u}}$ in the variational formulation (8), where $\boldsymbol{\lambda}$ is a Lagrange multiplier associated with zero mean velocity condition over $D_{1}$. Taking advantage of the linearity of the mapping $\boldsymbol{\lambda} \mapsto \mathbf{u}_{\boldsymbol{\lambda}}$, one just has to solve three generalized stokes problems, for instance for $\boldsymbol{\lambda}_{1}=(0,0), \boldsymbol{\lambda}_{2}=(1,0)$ and $\boldsymbol{\lambda}_{3}=(0,1)$, obtaining solutions $\mathbf{u}_{1}, \mathbf{u}_{2}$ and $\mathbf{u}_{3}$. The solution is then a convex combination of the three precomputed ones:

$$
\mathbf{u}=\alpha \mathbf{u}_{1}+\beta \mathbf{u}_{2}+(1-\alpha-\beta) \mathbf{u}_{3}
$$

where the coefficients $\alpha$ and $\beta$ can be computed by solving the $2 \times 2$ linear system

$$
\int_{D_{1}} \mathbf{u}=0 \Leftrightarrow \int_{D_{1}} \alpha \mathbf{u}_{1}+\beta \mathbf{u}_{2}+(1-\alpha-\beta) \mathbf{u}_{3}=0 \Leftrightarrow\left(\int_{D_{1}} \mathbf{u}_{1}-\mathbf{u}_{3}\right) \alpha+\left(\int_{D_{1}} \mathbf{u}_{2}-\mathbf{u}_{3}\right) \beta=-\int_{D_{1}} \mathbf{u}_{3} .
$$

Finally, we have to compute $B^{n+1}$ from $B^{n}$ and $\mathbf{u}^{n+1}$. In order to do that, as explained before, we use the real degree of freedom $\omega^{n}$ and write $\theta^{n+1}=\theta^{n}+\Delta t \omega^{n}$ where $\omega^{n}$ is computed from the velocity of the center of $D_{2}$ :

$$
\mathbf{V}^{n}=\frac{4}{\pi e^{2}} \int_{D_{2}} \mathbf{u}^{n} \quad \text { and } \quad \omega^{n}=\frac{\cos (\alpha) \mathbf{V}_{2}^{n}-\sin (\alpha) \mathbf{V}_{1}^{n}}{\ell}
$$

The geometric parameters used in our simulation are $h=20, r=20, \ell=14, \mu=1$ and $e=5$. The Navier-Stokes equations are written in dimensionless form using $h$ as the characteristic length and choosing the maximum mainstream velocity at the left boundary in such a way that the Reynolds number becomes $R e=U_{\max } /\left(\rho_{f} h\right)=400$.

The code was written in FreeFem++ version 1.44 and at each time step the generalized stokes problem is solved using standard finite elements. The source code can be downloaded from [1]. The initial velocity field was obtained by solving a Stokes problem. Figure 3 shows velocity fields and streamlines at different time steps. 

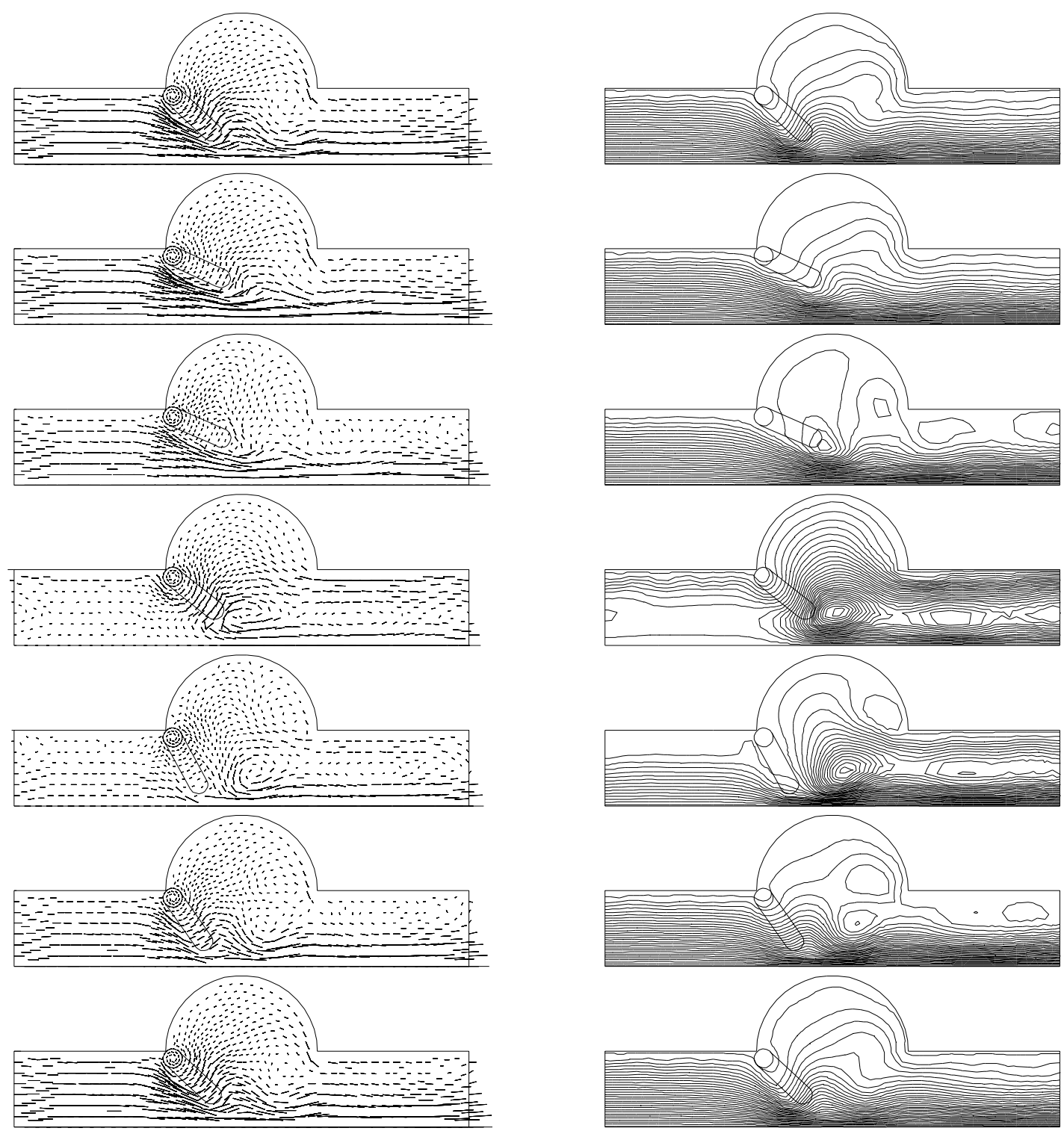

FiguRE 3. Velocity field and Streamlines at time steps 295-305-310-320-335-340-345

\section{REFERENCES}

[1] http://www.freefem.org, source code : http://www.freefem.org/examples/NSpenal.edp.

[2] V. Girault, P.-A. Raviart, Finite element methods for the Navier-Stokes equations, Springer, Berlin 1986 
[3] R. Glowinski, T.W. Pan, T.I. Hesla, D.D. Joseph, A distributed lagrange multiplier/fictitious domain method for particulate flow Int. J. of multiphase flow 25, 1998, pp. 755-794.

[4] H.H. Hu, Direct simulation of flows of solid-liquid mixtures Int. J. of Multiphase flow 22, 1996, pp. 335-352.

[5] A. A. Johnson, T. E. Tezduyar, Simulation of Multiple Spheres Falling in a Liquid-Filled Tube Computer Methods in Applied Mechanics and Engineering 134,pp. 351-373, 1996

[6] B. Maury, Optimisation sous Contrainte et Méthode des Éléments Finis, Notes de Cours. Université Paris-Sud (2005).

[7] B. Maury, Direct simulations of 2D fluid-particle flows in biperiodic domains Journal of computational physics 156, 1999, pp. 325-351.

[8] O.A. Oleinik, A.S. Shamaev, and G.A. Yosifian, Mathematical Problems in Elasticity and Homogenization North-Holland, Amsterdam, 1992.

[9] T.N. Randrianarivelo, G. Pianet, S. Vincent, J.P. Caltagirone, Numerical modelling of solid particle motion using a new penalty method, Int. J. Numer. Meth. Fluids 2005, 47, 1245-1251.

[10] F.K. Wippermann, On the fluid dynamics of the aortic valve Journal of Fluid Mechanics (1985), 159, pp. 487-501. 